\title{
Intrinsic coupling of orbital excitations to spin fluctuations in Mott insulators
}

\author{
Krzysztof Wohlfeld, ${ }^{1}$ Maria Daghofer, ${ }^{1}$ Satoshi Nishimoto, ${ }^{1}$ Giniyat Khaliullin, ${ }^{2}$ and Jeroen van den Brink ${ }^{1}$ \\ 1 IFW Dresden, P. O. Box 2701 16, D-01171 Dresden, Germany \\ ${ }^{2}$ Max-Planck-Institut für Festkörperforschung, Heisenbergstrasse 1, D-70569 Stuttgart, Germany
}

(Dated: June 9, 2022)

\begin{abstract}
We show how the general and basic asymmetry between two fundamental degrees of freedom present in strongly correlated oxides, spin and orbital, has very profound repercussions on the elementary spin and orbital excitations. Whereas the magnons remain largely unaffected, orbitons become inherently coupled with spin fluctuations in spin-orbital models with antiferromagnetic and ferroorbital ordered ground states. The composite orbiton-magnon modes that emerge fractionalize again in one dimension, giving rise to spin-orbital separation in the peculiar regime where spinons are faster than orbitons.
\end{abstract}

PACS numbers: 75.25.Dk, 75.30.Ds, 71.10.Fd, 74.72.Cj

In transition metal oxides, different $3 d$ orbitals near the Fermi level can have similar energy and thereby contribute to the low-energy physics. In presence of strong correlations charge fluctuations become suppressed and a Mott insulator is realized when there is a commensurate number of electrons per unit cell. The effective Hamiltonian that emerges, often referred to as Kugel-Khomskii (KK) Hamiltonian, can be expressed in terms of spin and orbital operators [1]. From a formal viewpoint the spin and orbital operators are very similar because they form identical algebras, but the way in which these operators enter into realistic KK Hamiltonians is very different.

While the spin wavefunction is in essence rotationally invariant and the coupling between spin operators therefore $S U(2)$ symmetric, the symmetry in orbital space is much lower due to the ubiquitous crystal field acting on the orbital wavefunctions in crystals. This reduced symmetry causes the orbital-orbital interaction to be very anisotropic in space and often inherently frustrated, which causes exotic effects such as macroscopic degeneracy of the groundstate or the emergence of nonAbelian topological excitations in the case of the compass or Kitaev models, respectively 2], which may be relevant for quantum computation 3, 4]. We will show that this asymmetry between spin and orbital degrees of freedom has fundamental repercussions on the coupling of the elementary spin and orbital excitations - the magnons and orbitons, respectively: whereas the magnons remain largely unaffected, orbitons become inherently coupled with spin fluctuations in spin-orbital models with antiferromagnetic $(\mathrm{AF})$ and ferroorbital $(\mathrm{FO})$ ordered ground states. This is relevant in the experimental context as substantial progress is being made in measuring orbitons [5, [6] and their dispersion, in particular in resonant inelastic x-ray scattering (RIXS) [7, 8], where we predict the coupling of the orbiton to magnetic fluctuations to be clearly discernible.

In the standard approach the complex problem of intertwined spin-orbital excitations is solved using a meanfield decoupling, i.e. considering magnons in a fixed or- bital background or orbitons in a fixed spin background. While such an approach can work well to obtain the correct spin and orbital orderings consistent with the Goodenough-Kanamori rules [1] and in some cases with ferromagnetic (FM) order [9], we show here that it fails to describe orbital excitations even qualitatively correctly for a number of spin-orbital models. We avoid this decoupling by mapping the coupled orbiton-magnon dynamics onto the well-controlled problem of a hole propagating in a magnetic background: the extensively studied single hole $t-J$ model. In particular, we find that in one dimension (1D) the orbital excitation fractionalizes into freely propagating spinon and orbiton, giving rise to spinorbital separation in the peculiar regime where spinons are faster than orbitons.

Model and problem statement. - The generic form of the KK Hamiltonian [1] in the Mott-insulating limit is

$$
\mathcal{H}=2 J\left(\sum_{\langle i, j\rangle} H_{i, j}^{S} H_{i j}^{T}+\sum_{i} H_{i}^{T}\right)
$$

where $i$ and $j$ are lattice sites and on each bond $\langle i, j\rangle$ the spin-spin interaction is $H_{i j}^{S}$ whereas the orbital-orbital one is $H_{i j}^{T}$. To capture the generic differences between orbitons and magnons, it is enough to break the $\mathrm{SU}(2)$ rotation symmetry in orbital space, which is here achieved by the large local crystal field breaking the degeneracy between the orbitals, expressed as $H_{i}^{T}=\frac{E_{z}}{2 J} T_{i}^{z}$. We keep for simplicity the rotational symmetry in the interactions so that for the spins $H_{i j}^{S}=\mathbf{S}_{i} \cdot \mathbf{S}_{j}+\frac{1}{4}$ and for the orbitals $H_{i j}^{T}=\mathbf{T}_{i} \cdot \mathbf{T}_{j}+\frac{1}{4}$, where $\mathbf{S}(\mathbf{T})$ are the spin (orbital) operators that fulfill the $S U(2)$ algebra for $S=1 / 2(T=1 / 2)$ spins (pseudospins). The constant $J>0$ gives the energy scale of the spin-orbital superexchange and the symmetry breaking field for the orbitals is $E_{z}$. Note that for the case of $E_{z}=0$ (not considered here), this model has an $S U(4)$ symmetry even higher than the combined $S U(2) \times S U(2)$ symmetries, which results in the ground state given by the Bethe Ansatz and composite spin-orbital gapless excitations in addition to the separate spin and orbital ones (see, e.g., Ref. [10]). 
This model describes the low-energy physics, determined by singly occupied sites, of a two-orbital Hubbard model in the limit of a large onsite Coulomb repulsion $U$ and vanishing Hund's exchange $J_{H}$, cf. Eqs. (1-5) and (29) in Ref. [9].

Here we are interested in the orbital excitations of the model Eq. (1) when $E_{z} \gg J$ i.e., the orbital splittings are larger than magnetic coupling energy. This is a realistic regime for many strongly correlated compounds such as 1D or two-dimensional (2D) cuprates [11]. As one degree of freedom is completely polarized, the ground state is easily found and given by all electrons occupying a single orbital in an AF state. But while decoupling spin and orbital degrees of freedom works here for the ground state, it is not at all appropriate for orbital excitations - this is the problem investigated below.

Decoupling of spin and orbital sector. - The ground state $|\psi\rangle=\left|\psi_{S}\right\rangle \otimes\left|\psi_{O}\right\rangle$ of Eq. (11) is described by the ground state $\left|\psi_{S}\right\rangle=|\mathrm{AF}\rangle$ of an AF Heisenberg system formed by spins in the lower-energy orbital, i.e., an FO ordered state $\left|\psi_{O}\right\rangle=|\mathrm{FO}\rangle$. The orbital excitations are reached by flipping an orbital, i.e., by promoting an electron at site $j$ from the occupied lower orbital to the empty higher band at the same site, expressed by the orbital raising operator $T_{j}^{+}$. The momentum-dependent orbital excitation is given by $T_{k}^{+}=\sum_{j} \mathrm{e}^{i k j} T_{j}^{+}\left[T_{k}^{-}=\left(T_{k}^{+}\right)^{\dagger}\right]$, and the spectral function describing its dynamics is

$$
O(k, \omega)=\frac{1}{\pi} \lim _{\eta \rightarrow 0} \Im\left\langle\psi\left|T_{k}^{-} \frac{1}{\omega+E_{\psi}-\mathcal{H}-i \eta} T_{k}^{+}\right| \psi\right\rangle .
$$

First we discuss the orbiton spectral function by taking the orbital excitation to be independent of the magnetic excitation. One can then rewrite the orbital operators by use of Holstein-Primakoff bosons (see, e.g., Ref. [12]), keep only quadratic terms in the expansion (orbital-wave theory) and, noticing that the ground state $\left|\psi_{O}\right\rangle$ does not contain bosons, obtain

$$
O(k, \omega)=\delta\left[\omega-\omega_{O W}(k)\right],
$$

with a mean-field orbital-wave dispersion $\omega_{O W}(k)=$ $E_{z}-\frac{1}{2} z J_{\mathrm{OW}}\left(1-\gamma_{k}\right)$. Here, $z$ is the coordination number, $\gamma_{k}$ is the lattice structure factor, and the effective orbital exchange constant $J_{\mathrm{OW}}=2 J\left\langle\psi_{S}\left|\mathbf{S}_{i} \cdot \mathbf{S}_{j}+\frac{1}{4}\right| \psi_{S}\right\rangle$. The orbital excitation on the mean-field level is thus a quasiparticle with a cosine-like dispersion with period $2 \pi$ : for example in 1D we obtain an effective reduced $J_{\mathrm{OW}} \simeq-0.4 J \ll J$, cf. the thick line in Fig. 2 An analogous procedure for magnons in FM planes with alternating orbitals $(\mathrm{AO})$ has been applied to $\mathrm{LaMnO}_{3}$ or $\mathrm{KCuF}_{3}$, and similarly yields magnons with a reduced bandwidth, but without any other trace of the AO order, in agreement with experiment 13. We will see, however, that for orbitons this framework of mean-field decoupling is greatly oversimplified.

Mapping onto an effective $t-J$ model. - The orbitalwave approximation puts all the impact of the AF order (a)

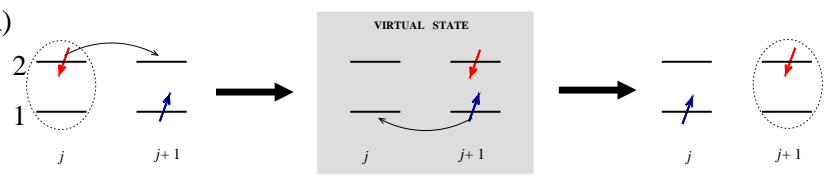

(b)

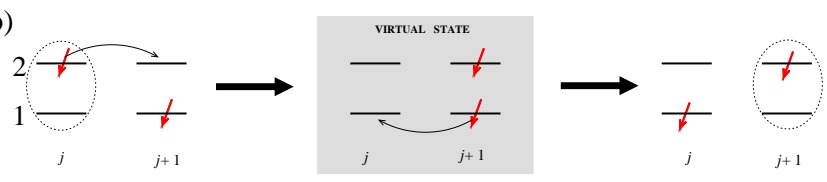

FIG. 1: (color online) Two superexchange processes moving an electron in an excited orbital (indicated by oval) from site $j$ to its neighbor $j+1$ : (a) and (b) describe orbiton motion when spins along the bond are antiparallel or parallel, respectively, see text. The states in the grey middle panels are not part of the low-energy Hilbert space corresponding to (1). These virtual excitations within the full two-orbital Hubbard model illustrate the origin of those superexchange interactions of Hamiltonian (1) that propagate the orbiton; note that the spin of the excited electron is conserved.

into $J_{\mathrm{OW}}$, which is a mean-field average of the sum of two distinct superexchange processes in which the orbital excitation may propagate through the lattice. The first one, which corresponds to $\left(T_{j}^{+} T_{j+1}^{-}+\right.$h.c. $)\left(S_{j}^{+} S_{j+1}^{-}+\right.$h.c. $)$ processes in Hamiltonian (1), allows for orbiton propagation when the spins on the bond are antiparallel, see Fig. 1(a). The second one, which corresponds to $\left(T_{j}^{+} T_{j+1}^{-}+\right.$h.c. $)\left(S_{j}^{z} S_{j+1}^{z}+1 / 4\right)$ processes in Hamiltonian (1), allows for orbiton propagation when the spins on the bond are parallel, see Fig. 1(b). Crucially (see next paragraph), this figure illustrates that in both cases the spin of the electron in the upper orbital 2 is conserved during the orbiton propagation. This is because spin-orbital Hamiltonian (10) is, as mentioned above, a low energy limit of the two-orbital Hubbard model with the Hund's exchange $J_{H}=0$ and the spins of individual electrons in the superexchange process cannot be flipped (see middle panels of Fig. 11). In more realistic spin-orbital models the Hund's exchange is finite [1], but the processes which would not conserve the electron's spin in the excited orbital are small $\left(\propto J_{H} / U\right)$ and thus could be neglected.

To be explicit, we now focus on the 1D case and employ a Jordan-Wigner transformation 14]. 2D and threedimensional (3D) cases are discussed afterwards. We thus introduce $S_{j}^{+}=\beta_{j} \mathrm{e}^{i \pi Q}, S_{j}^{-}=\mathrm{e}^{-i \pi Q} \beta_{j}^{\dagger}, S_{j}^{z}=$ $\frac{1}{2}-n_{j \beta}$, where $Q=\sum_{l=1}^{j-1} n_{l \beta}$ and $\beta_{j}^{\dagger}$ create spinons while $T_{j}^{+}=\mathrm{e}^{-i \pi \bar{Q}} \alpha_{j}^{\dagger}, T_{j}^{-}=\alpha_{j} \mathrm{e}^{i \pi \bar{Q}}, T_{j}^{z}=n_{j \alpha}-\frac{1}{2}$, where $\bar{Q}=\sum_{l=1}^{j-1} n_{l \alpha}$ and $\alpha_{j}^{\dagger}$ creates a pseudospinon. Since the spin of the propagating electron in the upper orbital 2 is conserved (see above), one may calculate the spectral function $O(k, \omega)$ for, e.g., spin-up in the upper orbital. We are then allowed to replace $T_{k}^{\dagger} \rightarrow \sum_{j} \mathrm{e}^{i k j} T_{j}^{+}\left(\frac{1}{2}+S_{j}^{z}\right)=\mathrm{e}^{-i \pi \bar{Q}} \alpha_{j}^{\dagger}\left(1-n_{j \beta}\right)$ in Eq. (2) and thus terms in the Hamiltonian that create or annihi- 
late both a spinon and a pseudospinon at the same site lead to a vanishing contribution to the spectral function. Phase factors cancel in one dimension and we obtain

$$
O(k, \omega)=\frac{1}{\pi} \lim _{\eta \rightarrow 0} \Im\left\langle\bar{\psi}\left|\alpha_{k} \frac{1}{\omega+E_{\bar{\psi}}-\overline{\mathcal{H}}-E_{z}-i \eta} \alpha_{k}^{+}\right| \bar{\psi}\right\rangle,
$$

with the effective fermionic Hamiltonian

$$
\begin{aligned}
\overline{\mathcal{H}}= & -\frac{1}{2} J \sum_{\langle i, j\rangle}\left(\beta_{i}^{\dagger} \alpha_{i} \alpha_{j}^{\dagger} \beta_{j}+\alpha_{i} \alpha_{j}^{\dagger}+\text { h.c. }\right) \\
& +J \sum_{\langle i, j\rangle}\left[\frac{1}{2}\left(\beta_{i}^{\dagger} \beta_{j}+\text { h.c. }\right)-\frac{1}{2}\left(n_{i \beta}+n_{j \beta}\right)+n_{i \beta} n_{j \beta}\right],
\end{aligned}
$$

and an implicit constraint $\forall_{j} \beta_{j}^{\dagger} \beta_{j}+\alpha_{j}^{\dagger} \alpha_{j} \leq 1$. Here $|\bar{\psi}\rangle$ is a tensor product of the magnetic ground state $\left|\psi_{S}\right\rangle=$ $|\mathrm{AF}\rangle$ expressed in terms of spinons and a vacuum state for pseudospinons [recall that we consider here a single orbiton only; this also allowed us to skip quartic terms in pseudospinons in Eq. (15)].

At this point, we observe that the resulting effective Hamiltonian is in fact a Hamiltonian for the $t-J$ model written in terms of the Jordan-Wigner fermions with the above constraint [15]. By introducing the electron operators $p_{j \uparrow}=\alpha_{j}^{\dagger}, \quad p_{j \downarrow}=\alpha_{j}^{\dagger} \beta_{j} \mathrm{e}^{i \pi Q}$ acting in the restricted Hilbert space without double occupancies we obtain

$$
O(k, \omega)=\frac{1}{\pi} \lim _{\eta \rightarrow 0} \Im\left\langle\tilde{\psi}\left|p_{k \uparrow}^{\dagger} \frac{1}{\omega+E_{\tilde{\psi}}-\tilde{\mathcal{H}}-E_{z}-i \eta} p_{k \uparrow}\right| \tilde{\psi}\right\rangle,
$$

with the $t-J$ Hamiltonian

$$
\tilde{\mathcal{H}}=-t \sum_{\langle i, j\rangle, \sigma}\left(p_{i \sigma}^{\dagger} p_{j \sigma}+h . c .\right)+J \sum_{\langle i, j\rangle}\left(\mathbf{S}_{i} \cdot \mathbf{S}_{j}+\frac{1}{4} n_{i} n_{j}\right),
$$

where $n_{j}=\sum_{\sigma} n_{j p \sigma}$ and the hopping parameter $t$ is defined as $t=J / 2[16]$. The ground state $|\tilde{\psi}\rangle$ is now the tensor product of a vacuum state for holes and the $\left|\psi_{S}\right\rangle$ state. We have thus mapped the single orbiton in the FO and AF chain, with dynamics governed by Hamiltonian (1), onto a single hole doped into the undoped AF chain with its dynamics governed by Hamiltonian (17).

Numerical results for the $t-J$ model. - To flesh out the resulting coupling between orbitons and spin fluctuations we use Lanczos exact diagonalization to evaluate Eq. (16) on a finite chain (28 sites). The spectral function is shown in Fig. 2, the spectrum differs qualitatively from the orbital-wave result shown as a thick line in Fig. 2, It now consists of multiple peaks (expected to merge into incoherent spectrum in the thermodynamic limit) instead of one single excitation. There is a dominant feature at the lower edge of the spectrum, but its periodicity is $\pi$ reflecting the doubled unit cell of the AF order.

The $t-J$ model with $J>t[16]$ is not easily accessible in the Hubbard type models, as it would formally

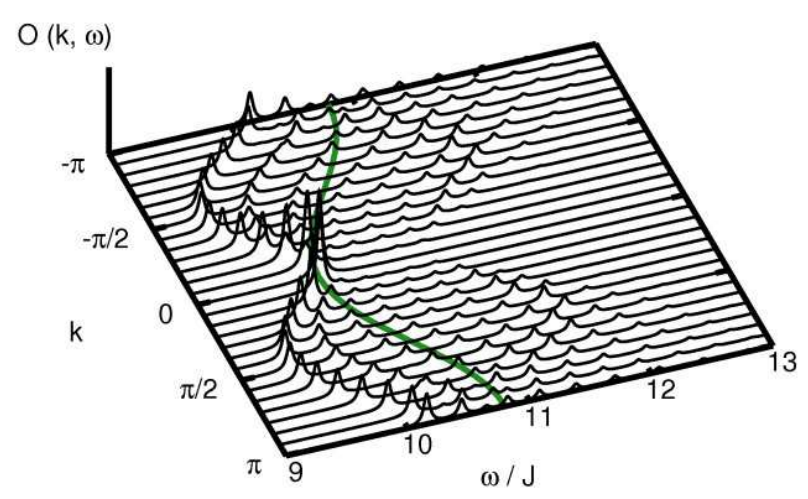

FIG. 2: (color online) Spectral function $O(k, \omega)$ of orbital excitation obtained via the mapping onto the $t-J$ model, Eq. (16), evaluated using Lanczos exact diagonalization on a 28 site chain. A broadening $\eta=0.03 J$ and $E_{z}=10 J$. The thick line shows orbital excitation in a mean-field (orbital-wave) approach, Eq. (3).

(a)

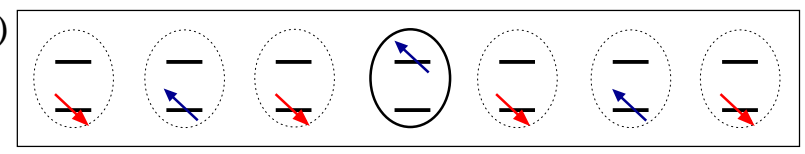

(b)

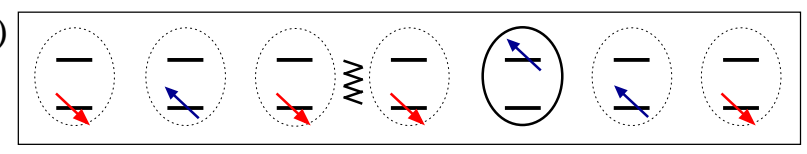

(c)

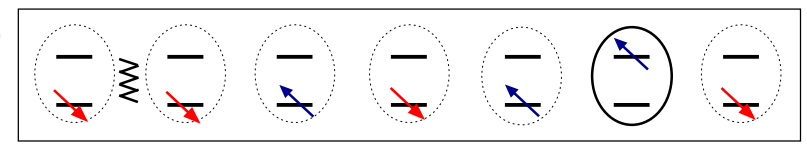

FIG. 3: (color online) Schematic representation of the orbital motion and the induced spin fluctuations giving rise to spinorbital separation in 1D. The first hop of the excited state $(\mathrm{a} \rightarrow \mathrm{b})$ creates a spinon (wavy line) that moves via spin exchange $\propto J$. Next hop $(\mathrm{b} \rightarrow \mathrm{c})$ does not produce any extra spinons: an 'orbiton' freely propagating as a 'holon' with an effective hopping $t \sim J / 2$ is created.

correspond to small onsite interaction $U$, where the $t$ $J$ model is no longer valid. In this regime, the spinon moves faster than the holon, and the entire lower edge of the spectrum is thus given by 'holon' states [17]. If the orbiton takes the place of the holon as argued here, this exotic behavior should be observable in RIXS experiments and one would expect a dominant excitation with orbiton-character with dispersion $\omega \approx E_{z}-2 t \sin |k|$ at the bottom of the spectrum. The latter would be expected to extend up to $\omega \approx E_{z}+\sqrt{J^{2}+4 t^{2}-4 t J \cos k}$ and to contain an intermediate feature still well-visible within the continuum with the dispersion of the purely 
orbiton-character scaling as $\omega \approx E_{z}+2 t \sin |k|$.

Figure 3 illustrates how electron exchange processes can let an orbital excitation propagate through the system after creating a spinon in the first step. The spinon itself moves via spin flips $\propto J>t$, faster than the orbiton, and the two get well separated. The orbital-wave picture, on the other hand, would require the orbital excitation to move without creating the spinon in the first step. As can be inferred from Fig. 3 this is only possible for imperfect Néel AF spin order so that the averages of processes shown in Fig. 1(a) and (b) are finite.

$2 D$ and $3 D$. - Remarkably, the standard OW picture becomes even worse in higher dimensions: in 2D (3D) cubic lattices, the mean-field orbital coupling $J_{\text {OW }}$ almost vanishes due to $\left\langle\psi_{S}\left|\mathbf{S}_{i} \cdot \mathbf{S}_{j}+\frac{1}{4}\right| \psi_{S}\right\rangle \simeq-0.08(-0.05)$; the orbital dynamics is then entirely governed by coupling to the spin fluctuations [18]. The $t-J$ model description of a single orbiton Eqs. (16-17) is general and valid for any dimension: the mapping rests entirely on the fact that spin on the excited orbital is conserved. In particular, the Jordan-Wigner fermionization applied to the 2D case gives the $t-J$ model expressed in terms of JordanWigner fermions 15 and consequently the 2D version of Eqs. (16[17), see [19] for details.

A very good approximate solution for the higherdimensional $t-J$ model with $J>t$ can be obtained by perturbation theory, because $J>t$ corresponds to weak coupling, see Eq. (7) of Ref. [20]. The solution shows that in $2 \mathrm{D}$ or $3 \mathrm{D}$ a single orbiton in the undoped AF system described by the $t-J$ model cannot fractionalize due the magnetic string effect. Still, the orbiton dressed with spin fluctuations is mobile on a renormalized scale [20]. For example the 2D case directly corresponds to the result in Ref. [21] which gives $\omega \approx E_{z}-x_{1}+x_{2}\left(\cos k_{x}+\cos k_{y}\right)^{2}+x_{3}\left(\cos 2 k_{x}+\cos 2 k_{y}\right)$ for the orbiton dispersion $\left(x_{i}\right.$ are positive parameters $\propto t^{2} / J$ when $J>t$, cf. Ref. [21] for exact values). We thus expect to observe, e.g. in high resolution RIXS experiment on $2 \mathrm{D}$ cuprates, a spectrum similar to the one-particle Greens function of the higher-dimensional $t-J$ model, with a low-energy quasi-particle orbiton peak $\propto \omega$ and additional incoherent part 21]. Crucially, while the parameters $x_{i}$ depend on $J / t$ and would vary in more realistic spin-orbital models [16], the general shape of the orbiton dispersion $\omega$ - the minimum at $(\pi / 2, \pi / 2)$, saddle point at $(\pi, 0)$, maxima at $(0,0)$ and $(\pi, \pi)$ - is robust reflecting the fact that the coherent motion of orbiton is possible only within a given spin-sublattice.

No analogue in spin sector. - Let us now revisit the analogue where the spin is polarized (FM) and the orbital sector shows AO order due to, e.g., a large Jahn-Teller effect. An example would be the FM and AO planes in $\mathrm{LaMnO}_{3}$ or $\mathrm{KCuF}_{3}[13$ ]. Many studies have shown that the orbital degrees of freedom merely renormalize the spin excitation in this case and do not change the periodicity of magnons (see, e.g., Ref. 22]). This qualitative difference is caused by the fact that in realistic cases the Jahn-Teller stabilized AO order is much more classical and robust, thus suppressing the creation of orbital excitations by the excited spin and giving larger weight to the pure spin excitation. In other words, spin waves are typically below the orbital gap and well protected by the underlying $S U(2)$ symmetry and Goldstone theorem.

In conclusion, we have shown that orbitons in realistic spin-orbital models with AF-spin and ferroorbital ground state are so strongly coupled to the spin excitations that the usual mean-field decoupling of two sectors breaks down. In fact, we have presented an exact mapping of the problem onto an effective $t-J$ model and have shown that the orbiton in such models behaves like a single hole in undoped AF Mott insulator. However, since the typical superexchange parameters for KK spin-orbital models lead to $J>t$, the study of orbiton problem provides access to a regime of the $t-J$ model which has been thought to be 'unphysical' in terms of single-band Hubbard models. Finally, in 1D signatures of spin-orbital separation are expected again in the peculiar regime where spinons are faster than orbitons.

We thank G. Jackeli, M.W. Haverkort and A.M. Oleś for discussions. Support from the Alexander von Humboldt Foundation (K.W.) and the DFG Emmy Noether Program (M.D.) is acknowledged.

[1] K.I. Kugel and D.I. Khomskii, Sov. Phys. Usp. 25, 231 (1982).

[2] G. Jackeli and G. Khaliullin, Phys. Rev. Lett. 102, 017205 (2009).

[3] A.Y. Kitaev, Annals of Physics 303, 2 (2003).

[4] B. Douçot, M. Feigel'man, L. Ioffe, and A. Ioselevich, Phys. Rev. B 71, 024505 (2005).

[5] C. Ulrich et al., Phys. Rev. Lett. 97, 157401 (2006).

[6] C. Ulrich et al., Phys. Rev. Lett. 103, 107205 (2009).

[7] A. Kotani and S. Shin, Rev. Mod. Phys. 73, 203 (2001).

[8] L.J.P. Ament et al., Rev. Mod. Phys. 83, 705 (2011).

[9] J. van den Brink et al., Phys. Rev. B 58, 10276 (1998).

[10] Y.-Q. Li, M. Ma, D.-N. Shi, and F.-C. Zhang, Phys. Rev. B 60, 12781 (1999).

[11] It turns out that the results below are valid also when the symmetry of the orbital superexchange term is further broken (as it may be the case in some compounds).

[12] K. Wohlfeld, A.M. Oleś, and P. Horsch, Phys. Rev. B 79, 224433 (2009).

[13] F. Moussa et al., Phys. Rev. B 54, 15149 (1996); B. Lake, D.A. Tennant, and S.E. Nagler, Phys. Rev. Lett. 85, 832 (2000).

[14] P. Jordan and E. Wigner, Z. Physik 47, 631 (1928).

[15] S. Barnes and S. Maekawa, Journal of Physics: Condensed Matter 14, L19 (2002).

[16] Note that inequivalent hoppings of orbitals [not included in model Eq. (10] would lead to $t \equiv 2 t_{1} t_{2} / U, J=4 t_{1}^{2} / U$ and $t \neq J / 2$, where $t_{1}\left(t_{2}\right)$ is the hopping of electrons in the lower (upper) orbital. Still $J>t$ holds since $t_{1} \geq t_{2}$.

[17] M. Brunner, F. Assaad, and A. Muramatsu, 
Eur. Phys. J. B 16, 209 (2000); H. Suzuura and N. Nagaosa, Phys. Rev. B 56, 3548 (1997).

[18] G. Khaliullin and S. Maekawa, Phys. Rev. Lett. 85, 3950 (2000).

[19] See supplementary material below for details of the derivation.

[20] S. Schmitt-Rink, C.M. Varma, and A.E. Ruckenstein, Phys. Rev. Lett. 60, 2793 (1988).

[21] G. Martinez and P. Horsch, Phys. Rev. B 44, 317 (1991).

[22] G. Khaliullin and S. Okamoto, Phys. Rev. B 68, 205109 (2003).

\section{SUPPLEMENTARY MATERIAL}

In what follows we show that the $t-J$ model description of the orbiton problem [Eqs. (6-7) in the main text] is not only valid in the $1 \mathrm{D}$ case but also in higher dimensions. To be explicit we concentrate now on the $2 \mathrm{D}$ case (from which the $3 \mathrm{D}$ case follows in a straightforward way) and introduce the Jordan-Wigner fermions $\alpha$ and $\beta$ for pseudospins and spins:

$$
\begin{aligned}
& S_{j}^{+}=\beta_{j} \mathrm{e}^{i \pi Q_{j}}, \\
& S_{j}^{-}=\mathrm{e}^{-i \pi Q_{j}} \beta_{j}^{\dagger}, \\
& S_{j}^{z}=\frac{1}{2}-n_{j \beta},
\end{aligned}
$$

where $Q_{j}=\sum_{l=1}^{j-1} n_{l \beta}$ and $\beta_{j}^{\dagger}$ create spinons while

$$
\begin{aligned}
& T_{j}^{+}=\mathrm{e}^{-i \pi \bar{Q}_{j}} \alpha_{j}^{\dagger}, \\
& T_{j}^{-}=\alpha_{j} \mathrm{e}^{i \pi \bar{Q}_{j}}, \\
& T_{j}^{z}=n_{j \alpha}-\frac{1}{2},
\end{aligned}
$$

where $\bar{Q}_{j}=\sum_{l=1}^{j-1} n_{l \alpha}$. Note that this is the same transformation as in the main text but, to keep track of the phase factors, we explicitly wrote the site index $j$ of the phase factors $Q_{j}$ and $\bar{Q}_{j}$.

Next, similarly as in $1 \mathrm{D}$, the spin of the propagating electron in the upper orbital 2 is conserved, and one may calculate the spectral function $O(k, \omega)$ for, e.g., spin-up in the upper orbital. We are then allowed to replace $T_{k}^{\dagger} \rightarrow \sum_{j} \mathrm{e}^{i k j} T_{j}^{+}\left(\frac{1}{2}+S_{j}^{z}\right)=\mathrm{e}^{-i \pi \bar{Q}_{j}} \alpha_{j}^{\dagger}\left(1-n_{j \beta}\right)$ in Eq. (2) in the main text and thus terms in the Hamiltonian that create or annihilate both a spinon and a pseudospinon at the same site also lead to a vanishing contribution to the spectral function.

In $2 \mathrm{D}$ one has to take care of the phase factors. However, the crucial observation is that for the case of the single orbiton the phase factors $\bar{Q}_{j}$ associated with a pseudospinon either do not contribute at all [Eq. (14) below] or cancel for the nearest neighbor bonds [Eq. (15) below] - similarly to 1D. Thus, these are only the spin phase factors $Q_{j}$ which are in the end present in the spectral function and Hamiltonian written in terms of the spinons and pseudospinons:

$$
O(k, \omega)=\frac{1}{\pi} \lim _{\eta \rightarrow 0} \Im\left\langle\bar{\psi}\left|\alpha_{k} \frac{1}{\omega+E_{\bar{\psi}}-\overline{\mathcal{H}}-E_{z}-i \eta} \alpha_{k}^{+}\right| \bar{\psi}\right\rangle,
$$

with the effective fermionic Hamiltonian

$$
\begin{gathered}
\overline{\mathcal{H}}=-\frac{1}{2} J \sum_{\langle i, j\rangle}\left(\mathrm{e}^{-i \pi Q_{i}} \beta_{i}^{\dagger} \alpha_{i} \alpha_{j}^{\dagger} \beta_{j} \mathrm{e}^{i \pi Q_{j}}+\alpha_{i} \alpha_{j}^{\dagger}+h . c .\right) \\
+J \sum_{\langle i, j\rangle}\left[\frac{1}{2}\left(\mathrm{e}^{-i \pi Q_{i}} \beta_{i}^{\dagger} \beta_{j} \mathrm{e}^{i \pi Q_{j}}+\text { h.c. }\right)\right. \\
\left.-\frac{1}{2}\left(n_{i \beta}+n_{j \beta}\right)+n_{i \beta} n_{j \beta}\right] .
\end{gathered}
$$

As stated above (and mentioned for the 1D case in the main text), here we have an implicit constraint $\forall_{j} \beta_{j}^{\dagger} \beta_{j}+$ $\alpha_{j}^{\dagger} \alpha_{j} \leq 1$ while $|\bar{\psi}\rangle$ is a tensor product of the magnetic ground state $\left|\psi_{S}\right\rangle=|\mathrm{AF}\rangle$ expressed in terms of spinons and a vacuum state for pseudospinons.

At this point, we observe that the resulting effective Hamiltonian is in fact a Hamiltonian for the $t-J$ model written in terms of the Jordan-Wigner fermions with the above constraint. By introducing the electron operators $p_{j \uparrow}=\alpha_{j}^{\dagger}, \quad p_{j \downarrow}=\alpha_{j}^{\dagger} \beta_{j} \mathrm{e}^{i \pi Q_{j}}$ acting in the restricted Hilbert space without double occupancies we obtain

$$
O(k, \omega)=\frac{1}{\pi} \lim _{\eta \rightarrow 0} \Im\left\langle\tilde{\psi}\left|p_{k \uparrow}^{\dagger} \frac{1}{\omega+E_{\tilde{\psi}}-\tilde{\mathcal{H}}-E_{z}-i \eta} p_{k \uparrow}\right| \tilde{\psi}\right\rangle,
$$

with the $t-J$ Hamiltonian

$$
\tilde{\mathcal{H}}=-t \sum_{\langle i, j\rangle, \sigma}\left(p_{i \sigma}^{\dagger} p_{j \sigma}+h . c .\right)+J \sum_{\langle i, j\rangle}\left(\mathbf{S}_{i} \cdot \mathbf{S}_{j}+\frac{1}{4} n_{i} n_{j}\right),
$$

where $n_{j}=\sum_{\sigma} n_{j p \sigma}$ and the hopping parameter $t$ is defined as $t=J / 2$. The ground state $|\tilde{\psi}\rangle$ is now the tensor product of a vacuum state for holes and the $\left|\psi_{S}\right\rangle$ state. We have thus mapped the problem of a single orbital excitation in the 2D FO and AF ground state, with dynamics governed by Hamiltonian Eq. (1) in the main text, onto a single hole doped into the undoped 2D AF ground state with its dynamics governed by Hamiltonian (17). 